PROCEEDINGS OF THE

AMERICAN MATHEMATICAL SOCIETY

Volume 137, Number 4, April 2009, Pages 1439-1445

S 0002-9939(08)09718-9

Article electronically published on November 3, 2008

\title{
AFFINE INTERVAL EXCHANGE TRANSFORMATIONS WITH FLIPS AND WANDERING INTERVALS
}

\author{
C. GUTIERREZ, S. LLOYD, AND B. PIRES
}

(Communicated by Jane M. Hawkins)

\begin{abstract}
There exist uniquely ergodic affine interval exchange transformations of $[0,1]$ with flips which have wandering intervals and are such that the support of the invariant measure is a Cantor set.
\end{abstract}

\section{INTRODUCTION}

Let $N$ be a compact subinterval of either $\mathbb{R}$ or the circle $S^{1}$, and let $f: N \rightarrow N$ be piecewise continuous. We say that a subinterval $J \subset N$ is a wandering interval of the map $f$ if the forward iterates $f^{n}(J), n=0,1,2, \ldots$, are pairwise disjoint intervals, each not reduced to a point, and the $\omega$-limit set of $J$ is an infinite set.

A great deal of information about the topological dynamics of a map $f: N \rightarrow N$ is revealed when one knows whether $f$ has wandering intervals. This turns out to be a subtle question whose answer depends on both the topological and regularity properties of the map $f$.

The question of the existence of wandering intervals first arose in the case where $f$ is a diffeomorphism of the circle $S^{1}$. The Denjoy counterexample shows that even a $C^{1}$ diffeomorphism $f: S^{1} \rightarrow S^{1}$ may have wandering intervals. This behaviour is ruled out when $f$ is smoother. More specifically, if $f$ is a $C^{1}$ diffeomorphism of the circle such that the logarithm of its derivative has bounded variation, then $f$ has no wandering intervals [6]. In this case the topological dynamics of $f$ is simple: if $f$ has no periodic points, then $f$ is topologically conjugate to a rotation.

The first results ensuring the absence of wandering intervals on continuous maps satisfying some smoothness conditions were provided by Guckenheimer [8], Yoccoz [19, and Blokh and Lyubich 2. Later on, de Melo et al. 13] generalised these results, proving that if $N$ is compact and $f: N \rightarrow N$ is a $C^{2}$ map with non-flat critical points, then $f$ has no wandering intervals. Concerning discontinuous maps, Berry and Mestel [1] found a condition which excludes wandering intervals in Lorenz maps - interval maps with a single discontinuity. Of course, conservative maps and, in particular, interval exchange transformations admit no wandering intervals. We consider the following generalisation of interval exchange transformations.

Let $0 \leq a<b$ and let $\{a, b\} \subset D \subset[a, b]$ be a discrete set consisting of $n+1$ points. We say that an injective, continuously differentiable map $T:[a, b] \rightarrow[a, b]$

Received by the editors February 28, 2008, and, revised form, May 31, 2008.

2000 Mathematics Subject Classification. Primary 37E05, 37E10; Secondary 37Bxx.

The first author was partially supported by FAPESP Grant 03/03107-9 and by CNPq Grants 470957/2006-9 and 306328/2006-2.

(C)2008 American Mathematical Society
Reverts to public domain 28 years from publication 1439 
defined on $\mathcal{D}(T)=[a, b] \backslash D$ is an affine interval exchange transformation of $n$ subintervals, or $n$-AIET for short, if $|D T|$ is a positive, locally constant function such that $T([a, b] \backslash D)$ is all of $[a, b]$ except for finitely many points. We also assume that the points in $D \backslash\{a, b\}$ are non-removable discontinuities of $T$. We say that an AIET is oriented if $D T>0$; otherwise we say that $T$ has flips. An isometric IET of $n$ subintervals, or $n$-IET for short, is an $n$-AIET satisfying $|D T|=1$ everywhere.

Levitt 11] found an example of a non-uniquely ergodic oriented AIET with wandering intervals. Gutierrez and Camelier [4] constructed an AIET with wandering intervals that is semiconjugate to a self-similar IET. The regularity of conjugacies between AIETs and self-similar IETs was examined by Cobo [5] and by Liousse and Marzougui 12. Recently, Bressaud, Hubert and Maass 3 provided sufficient conditions for a self-similar IET to have an AIET with a wandering interval semiconjugate to it.

In this paper we present an example of a self-similar IET with flips having the particular property that we can apply the main result of the work [3] to obtain a 5-AIET with flips that is semiconjugate to the IET and has densely distributed wandering intervals. The AIET so obtained is uniquely ergodic [17] (see [14, 18), and the support of the invariant measure is a Cantor set.

A few remarks are due in order to place this example in context. The existence of minimal non-uniquely ergodic AIETs with flips and wandering intervals would follow by the argument of Levitt [11, provided we know a minimal non-uniquely ergodic IET with flips. However, no example of a minimal non-uniquely ergodic IET with flips is known, although it is possible to insert flips in the example of Keane [10. (for oriented IETs) to get a transitive non-uniquely IET with flips having saddle-connections. Computational evaluations indicate that it is impossible to obtain, via Rauzy induction, examples of self-similar 4-IETs with flips that meet the hypotheses of [3], despite this being possible in the case of oriented 4-IETs (see [4, 5]). Thus, the example we present here is the simplest possible in the sense that wandering intervals do not occur for AIETs with flips that are semiconjugate to a self-similar IET, obtained via Rauzy induction, defined on a smaller number of intervals.

\section{SElF-SIMIlAR INTERVAL EXCHANGE TRANSFORMATIONS}

Let $T:[a, b] \rightarrow[a, b]$ be an $n$-AIET defined on $[a, b] \backslash D$, where $D=\left\{x_{0}, \ldots, x_{n}\right\}$ and $a=x_{0}<x_{1}<\ldots<x_{n-1}<x_{n}=b$. Let $\beta_{i} \neq 0$ be the derivative of $T$ on $\left(x_{i-1}, x_{i}\right), i=1,2 \ldots, n$. We shall refer to

$$
x=\left(x_{0}, x_{1}, \ldots, x_{n}\right)
$$

as the $D$-vector of $T$ (i.e. the domain-of-definition-vector of $T$ ). The vectors

$$
\gamma=\left(\log \left|\beta_{1}\right|, \log \left|\beta_{2}\right|, \ldots, \log \left|\beta_{n}\right|\right) \quad \text { and } \quad \tau=\left(\frac{\beta_{1}}{\left|\beta_{1}\right|}, \frac{\beta_{2}}{\left|\beta_{2}\right|}, \ldots, \frac{\beta_{n}}{\left|\beta_{n}\right|}\right)
$$

will be called the log-slope-vector and the flips-vector of $T$, respectively. Notice that $T$ has flips if and only if some coordinate of $\tau$ is equal to -1 . Let

$$
\left\{z_{1}, \ldots, z_{n}\right\}=\left\{T\left(\frac{x_{0}+x_{1}}{2}\right), T\left(\frac{x_{1}+x_{2}}{2}\right), \ldots, T\left(\frac{x_{n-1}+x_{n}}{2}\right)\right\}
$$


be such that $0<z_{1}<z_{2}<\ldots<z_{n}<1$; we define the permutation $\pi$ associated to $T$ as the one that takes $i \in\{1,2, \ldots, n\}$ to $\pi(i)=j$ if and only if $z_{j}=T\left(\left(x_{i-1}+\right.\right.$ $\left.\left.x_{i}\right) / 2\right)$.

It should be remarked that an AIET $T:[a, b] \rightarrow[a, b]$ with flips-vector $\tau \in$ $\{-1,1\}^{n}$ which has the zero vector as the log-slope-vector is an IET (with flipsvector $\tau$ ), and conversely. Let $J=[c, d]$ be a proper subinterval of $[a, b]$. We say that the IET $E$ is self-similar (on $J$ ) if there exists an orientation preserving affine map $L: \mathbb{R} \rightarrow \mathbb{R}$ such that $L(J)=[a, b]$ and $L \circ \widetilde{E}=E \circ L$, where $\widetilde{E}: J \rightarrow J$ denotes the IET induced by $E$ and $L(\mathcal{D}(\widetilde{E})) \subset \mathcal{D}(E)$. A self-similar IET $E:[a, b] \rightarrow[a, b]$ on a proper subinterval $J \subset[a, b]$ will be denoted by $(E, J)$.

Given an AIET $T:[a, b] \rightarrow[a, b]$, the orbit of $p \in[a, b]$ is the set

$$
O(p)=\left\{T^{n}(p) \mid n \in \mathbb{Z} \text { and } p \in \mathcal{D}(T)\right\} .
$$

The AIET $T$ is called transitive if there exists an orbit of $T$ that is dense in $[a, b]$. We say that the orbit of $p \in[a, b]$ is finite if $\#(O(p))<\infty$. In this way, a point $p \in[a, b]-\left(\mathcal{D}(T) \cup \mathcal{D}\left(T^{-1}\right)\right)$ has a finite orbit $O(p)=\{p\}$. A transitive AIET is minimal if it has no finite orbits.

Let $E:[a, b] \rightarrow[a, b]$ be an IET with D-vector $\left(x_{0}, x_{1}, \cdots, x_{n}\right)$. Denote by $J=[c, d]$ a proper subinterval of $[a, b]$. Suppose that $E$ is self-similar (on $J$ ); then there exists an IET $\widetilde{E}: J \rightarrow J$ such that $L(J)=[a, b]$ and $L \circ \widetilde{E}=E \circ L$. For $i=0,1, \cdots, n$, let $y_{i}=L^{-1}\left(x_{i}\right)$. Thus, the sequence of discontinuities of $\widetilde{E}$ is $\left\{y_{1}, \cdots, y_{n-1}\right\}$.

We say that a non-negative matrix is eventually positive if some power of it is a positive matrix. A non-negative matrix is eventually positive if and only if it is both irreducible and aperiodic. Let $A$ be an $n \times n$ non-negative matrix whose entries are:

$$
A_{j i}=\#\left\{0 \leq k \leq N_{i}: E^{k}\left(\left(y_{i-1}, y_{i}\right)\right) \subset\left(x_{j-1}, x_{j}\right)\right\},
$$

where $N_{i}$ is the smallest non-negative integer such that for some $y \in\left(y_{i-1}, y_{i}\right)$ (and therefore for all $\left.y \in\left(y_{i-1}, y_{i}\right)\right), E^{N_{i}+1}(y) \in J$. We shall refer to $A$ as the matrix associated to $(E, J)$. Being self-similar, $E$ is also transitive, which implies that $A$ is eventually positive. Hence, by the Perron-Frobenius Theorem [7, $A$ possesses exactly one probability right eigenvector $\alpha \in \Lambda_{n}$, where

$$
\Lambda_{n}=\left\{\lambda=\left(\lambda_{1}, \ldots, \lambda_{n}\right) \mid \lambda_{i}>0 \forall i\right\} .
$$

Moreover, the eigenvalue $\mu$ corresponding to $\alpha$ is simple, real and greater than 1; also, all other eigenvalues of $A$ have absolute value less than $\mu$. It was proved by Veech [17] (see also [14, 18]) that every self-similar IET is minimal and uniquely ergodic. Furthermore, following Rauzy [16], we conclude that

$$
\alpha=\left(x_{1}-x_{0}, x_{2}-x_{1}, \cdots, x_{n}-x_{n-1}\right) .
$$

\section{The theorem of Bressaud, Hubert and MaAss}

Let $A \in S L_{n}(\mathbb{Z})$ and let $\mathbb{Q}[t]$ be the ring of polynomials with rational coefficients in one variable. We say that two real eigenvalues $\theta_{1}$ and $\theta_{2}$ of $A$ are conjugate if there exists an irreducible polynomial $f \in \mathbb{Q}[t]$ such that $f\left(\theta_{1}\right)=f\left(\theta_{2}\right)=0$. We say that an AIET $T$ of $[0,1]$ is semiconjugate (resp. conjugate) to an IET $E$ of $[0,1]$ if there exists a non-decreasing (resp. bijective) continuous map $h:[0,1] \rightarrow[0,1]$ such that $h(\mathcal{D}(T)) \subset \mathcal{D}(E)$ and $E \circ h=h \circ T$. 
Theorem 1 (Bressaud, Hubert and Maass, 2007). Let $J$ be a proper subinterval of $[0,1]$, let $E:[0,1] \rightarrow[0,1]$ be an interval exchange transformation which is selfsimilar on $J$, and let $A$ be the matrix associated to $(E, J)$. Let $\theta_{1}$ be the PerronFrobenius eigenvalue of $A$. Assume that $A$ has a real eigenvalue $\theta_{2}$ such that

(1) $1<\theta_{2}\left(<\theta_{1}\right)$;

(2) $\theta_{1}$ and $\theta_{2}$ are conjugate.

Then there exists an affine interval exchange transformation $T$ of $[0,1]$ with wandering intervals that is semiconjugate to $E$.

Proof. This theorem was proved in [3] for oriented IETs. The same proof holds word for word for IETs with flips. In this case, the AIET $T$ inherits its flips from the IET $E$ through the previously constructed semiconjugacy.

\section{The interval EXChANGe tRANSFORMATION $E$}

In this section we shall present the IET we shall use to construct the AIET with flips and wandering intervals. We shall need the Rauzy induction 16, 15, 9] to obtain a minimal, self-similar IET whose associated matrix satisfies all the hypotheses of Theorem 1.

Let $\alpha=\left(\alpha_{1}, \alpha_{2}, \alpha_{3}, \alpha_{4}, \alpha_{5}\right) \in \Lambda_{5}$ be the probability Perron-Frobenius right eigenvector of the matrix

$$
A=\left(\begin{array}{lllll}
2 & 4 & 6 & 5 & 2 \\
0 & 2 & 1 & 1 & 1 \\
0 & 0 & 3 & 2 & 0 \\
1 & 2 & 2 & 2 & 1 \\
1 & 3 & 5 & 4 & 2
\end{array}\right)
$$

The eigenvalues $\theta_{1}, \theta_{2}, \rho_{1}, \rho_{2}, \rho_{3}$ of $A$ are real and have approximate values

$$
\theta_{1}=7.829, \theta_{2}=1.588, \rho_{1}=1, \rho_{2}=0.358, \rho_{3}=0.225
$$

and $\alpha=\left(\alpha_{1}, \alpha_{2}, \alpha_{3}, \alpha_{4}, \alpha_{5}\right)$, the probability right eigenvector associated to $\theta_{1}$, has approximate value

$$
\alpha=(0.380,0.091,0.070,0.170,0.289) .
$$

In what follows we represent a permutation $\pi$ of the set $\{1,2, \ldots, n\}$ by the $n$-tuple $\pi=(\pi(1), \pi(2), \ldots, \pi(n))$.

We consider the IET $E:[0,1] \rightarrow[0,1]$, which is determined by the following conditions:

(1) $E$ has the D-vector $x=\left(x_{0}, x_{1}, x_{2}, x_{3}, x_{4}, x_{5}\right)$, where

$$
x_{0}=0, \quad x_{i}=\sum_{k=1}^{i} \alpha_{k} \text { for } i=1, \ldots, 5 ;
$$

(2) $E$ has associated permutation $(5,3,2,1,4)$;

(3) $E$ has flips-vector $(-1,-1,1,1,-1)$.

Lemma 2. The map $E$ is self-similar on the interval $J=\left[0,1 / \theta_{1}\right]$, and $A$ is precisely the matrix associated to $(E, J)$. 
Proof. We apply the Rauzy algorithm to the IET $E$. We represent $E: I \rightarrow I$ by the pair $E^{(0)}=\left(\alpha^{(0)}, p^{(0)}\right)$, where $\alpha^{(0)}=\alpha$ is its length vector and $p^{(0)}=$ $(-5,-3,2,1,-4)$ is its signed permutation obtained by elementwise multiplication of the permutation $(5,3,2,1,4)$ with the flips-vector $(-1,-1,1,1,-1)$. We shall apply the Rauzy procedure fourteen times, obtaining IETs $E^{(k)}=\left(\alpha^{(k)}, p^{(k)}\right)$, $k=0, \ldots, 14$, with D-vector $x^{(k)}$ given by $x_{0}^{(k)}=0$ and $x_{i}^{(k)}=\sum_{j=1}^{i} \alpha_{j}^{(k)}$ for $i=1,2, \ldots, 5$.

\begin{tabular}{|c|rrrrr|c|}
\hline$k$ & \multicolumn{5}{|c|}{$p^{(k)}$} & $t^{(k)}$ \\
\hline 0 & -5 & -3 & 2 & 1 & -4 & 1 \\
1 & 4 & -5 & -3 & 2 & 1 & 0 \\
2 & 5 & -2 & -4 & 3 & 1 & 1 \\
3 & 5 & 1 & -2 & -4 & 3 & 1 \\
4 & 5 & 3 & 1 & -2 & -4 & 1 \\
5 & 5 & -4 & 3 & 1 & -2 & 0 \\
6 & -2 & -5 & 4 & 1 & -3 & 1 \\
7 & -2 & 3 & -5 & 4 & 1 & 0 \\
8 & -3 & 4 & -2 & 5 & 1 & 1 \\
9 & -3 & 4 & -2 & 5 & 1 & 1 \\
10 & -3 & 4 & -2 & 5 & 1 & 0 \\
11 & -4 & 5 & -3 & 2 & 1 & 1 \\
12 & -4 & 5 & 1 & -3 & 2 & 1 \\
13 & -4 & 5 & 2 & 1 & -3 & 0 \\
14 & -5 & -3 & 2 & 1 & -4 & 1 \\
\hline
\end{tabular}

TABLE 1. Rauzy cycle with associated matrix $A$.

Given an IET $E^{(k)}$, defined on an interval $\left[0, L^{(k)}\right]$ and represented by the pair $\left(\alpha^{(k)}, p^{(k)}\right)$, the IET $E^{(k+1)}$ is defined to be the map induced on the interval $\left[0, L^{(k+1)}\right]$ by $E^{(k)}$, where $L^{(k+1)}=L^{(k)}-\min \left\{\alpha_{5}^{(k)}, \alpha_{s}^{(k)}\right\}$ and $s$ is such that $\left|p_{n}^{(k)}(s)\right|=5$. We say that the type $t^{(k)}$ of $E^{(k)}$ is 0 if $\alpha_{5}^{(k)}>\alpha_{s}^{(k)}$ and 1 if $\alpha_{5}^{(k)}<\alpha_{s}^{(k)}$. Notice that $\sum_{i=1}^{5} \alpha_{i}^{(k)}=L^{(k)}$.

The new signed permutations $p^{(k)}$ obtained by this procedure are given in Table1, along with the type $t^{(k)}$ of $E^{(k)}$. The length vector $\alpha^{(k+1)}$ is obtained from $\alpha^{(k)}$ by the equation $\alpha^{(k)}=M\left(p^{(k)}, t^{(k)}\right) \cdot \alpha^{(k+1)}$, where $M\left(p^{(k)}, t^{(k)}\right) \in S L_{n}(\mathbb{Z})$ is a certain elementary matrix (see [9]). Moreover, we have that

$$
M\left(p^{(0)}, t^{(0)}\right) \cdots M\left(p^{(13)}, t^{(13)}\right)=A .
$$

Thus $\alpha^{(14)}=A^{-1} \cdot \alpha^{(0)}=\alpha^{(0)} / \theta_{1}$, and $J=\left[0, L^{(14)}\right]$. Notice that $p^{(14)}=p^{(0)}$, so we have a Rauzy cycle: $E^{(14)}$ and $E^{(0)}$ have the same flips-vector and permutation. Hence $\widetilde{E}=E^{(14)}$ is a $1 / \theta_{1}$-scaled copy of $E=E^{(0)}$, and so $E$ is self-similar on the interval $J$.

As remarked earlier, since $E$ self-similar, we have that the matrix associated to $(E, J)$ is eventually positive. In fact, we have that $A$ is the matrix associated to $(E, J)$. To see this, for $i \in\{0, \ldots, 5\}$ let $y_{i}=x_{i} / \theta_{1}$ be the points of discontinuity for $\widetilde{E}$. Table 2 shows the itinerary $I(i)=\left\{I(i)_{k}\right\}_{k=1}^{N_{i}}$ of each interval 
$\left(y_{i-1}, y_{i}\right)$, where $N_{i}=\min \left\{n>1: E^{n+1}\left(\left(y_{i-1}, y_{i}\right)\right) \subset J\right\}$ and $I(i)_{k}=r$ if and only if $E^{k}\left(\left(y_{i-1}, y_{i}\right)\right) \subset\left(x_{r-1}, x_{r}\right)$.

\begin{tabular}{|c|c|l|}
\hline$i$ & $N_{i}$ & \multicolumn{1}{|c|}{$I(i)$} \\
\hline 1 & 4 & 1514 \\
2 & 11 & 15214152154 \\
3 & 17 & 15214153153153154 \\
4 & 14 & 15214153153154 \\
5 & 6 & 152154 \\
\hline
\end{tabular}

TABLE 2. Itineraries $I(i), i \in\{1, \ldots, 5\}$.

The number of times that $j$ occurs in $I(i)$, for $i, j \in\{1, \ldots, 5\}$, is precisely $A_{j i}$; thus $A$ is the matrix associated to the pair $(E, J)$, as required.

Theorem A. There exists a uniquely ergodic affine interval exchange transformation of $[0,1]$ with flips that has wandering intervals and is such that the support of the invariant measure is a Cantor set.

Proof. By construction, the matrix $A$ associated to $(E, J)$ satisfies hypothesis (1) of Theorem 1 The characteristic polynomial $p(t)$ of $A$ can be written as the product of two irreducible polynomials over $\mathbb{Q}[t]$ :

$$
p(t)=(1-t)\left(1-8 t+18 t^{2}-10 t^{3}+t^{4}\right) .
$$

Thus the eigenvalues $\theta_{1}$ and $\theta_{2}$ are zeros of the same irreducible polynomial of degree four and so are conjugate. Hence, $A$ also satisfies hypothesis (2) of Theorem 1 . which finishes the proof.

Note that for an AIET $T$, the forward and backward iterates of a wandering interval $J$ form a pairwise disjoint collection of intervals. Moreover, when $T$ is semiconjugate to a transitive IET, as is the case in Theorem A, the $\alpha$-limit set and $\omega$-limit set of $J$ coincide.

\section{REFERENCES}

1. D. Berry and B. D. Mestel. Wandering intervals for Lorenz maps with bounded nonlinearity. Bull. London Math. Soc. 23 (1991), 183-189. MR.1122907 (93c:58119)

2. A. M. Blokh and M. Yu. Lyubich. Non-existence of wandering intervals and structure of topological attractors of one-dimensional dynamical systems, II. The smooth case. Ergod. Th. and Dyn. Sys. 9 (1989), 751-758. MR1036906 (91e:58101)

3. X. Bressaud, P. Hubert and A. Maass. Persistence of wandering intervals in self-similar affine interval exchange transformations. Preprint, arXiv:math.DS/08012088 (2007).

4. R. Camelier and C. Gutierrez. Affine interval exchange transformations with wandering intervals. Ergod. Th. and Dyn. Sys. 17 (1997), no. 6, 1315-1338. MR1488320 (99e:58117)

5. M. Cobo. Piece-wise affine maps conjugate to interval exchanges. Ergod. Th. and Dyn. Sys. 22 (2002), no. 2, 375-407. MR1898797 (2003h:37003)

6. A. Denjoy. Sur le courbes définies par les équations différentielles à la surface du tore. J. Math. Pure et Appl. 11 (1932), série 9, 333-375.

7. F. R. Gantmacher. Applications of the Theory of Matrices. Interscience, New York (1959). MR0107648(21:6372b)

8. J. Guckenheimer. Sensitive dependence to initial conditions for one-dimensional maps. Commun. Math. Phys. 70 (1979), 133-160. MR.553966 (82c:58037) 
9. C. Gutierrez, S. Lloyd, V. Medvedev, B. Pires and E. Zhuzhoma. Unique ergodicity of circle and interval exchange transformations with flips. Preprint, arXiv:math.DS/07113821 (2007).

10. M. Keane. Non-ergodic interval exchange transformations. Israel J. Math. 26 (1977), no. 2, 188-196. MR0435353 (55:8313)

11. G. Levitt. La décomposition dynamique et la différentiabilité des feuilletages des surfaces. Ann. Inst. Fourier (Grenoble) 37 (1987), 85-116. MR916275 (88m:57035)

12. I. Liousse and H. Marzougui. Échanges d'intervalles affines conjugués à des linéaires. Ergod. Th. and Dyn. Sys. 22 (2002), no. 2, 535-554. MR1898804(2003c:37050)

13. M. Martens, W. de Melo and S. van Strien. Julia-Fatou-Sullivan theory for real onedimensional dynamics. Acta Math. 168 (1992), 273-318. MR1161268 (93d:58137)

14. H. Masur. Interval exchange transformations and measured foliations. Ann. of Math. (2) 115 (1982), 169-200. MR644018 (83e:28012)

15. A. Nogueira. Almost all interval exchange transformations with flips are nonergodic. Ergod. Th. and Dyn. Sys. 9 (1989), no. 3, 515-525. MR1016669 (91d:28035)

16. G. Rauzy. Échanges d'intervalles et transformations induites. Acta Arith. 34 (1979), 315-328. MR:543205 (82m:10076)

17. W. Veech. Interval exchange transformations. J. d'Analyse Math. 33 (1978), 222-272. MR $516048(80 \mathrm{e}: 28034)$

18. W. Veech. Gauss measures for transformations on the space of interval exchange maps. Ann. of Math. (2) 115 (1982), 201-242. MR644019 (83g:28036b)

19. Y. C. Yoccoz. Il n'y a pas de contre-exemple de Denjoy analytique. C. R. Acad. Sci. Paris Sér. I Math. 298 (1984), no. 7, 141-144. MR741080(85j:58134)

Instituto de Ciências Matemáticas e de ComputaÇão, Universidade de São Paulo, São CARlos - SP, Brazil

E-mail address: gutp@icmc.usp.br

School of Mathematics and Statistics, University of New South Wales, Sydney, NSW, Australia

E-mail address: s.lloyd@unsw.edu.au

Departamento de Física e Matemática, Faculdade de Filosofia, Ciências e Letras da Universidade de São Paulo, Ribeirão Preto - SP, Brazil

E-mail address: benito@ffclrp.usp.br 